\title{
Interval-Valued Fuzzy Ideals of a Ring
}

\author{
Keon Chang Lee ${ }^{1}$, Kul Hur ${ }^{2}$ and Pyung Ki Lim ${ }^{3}$ \\ ${ }^{1}$ Department of Computer Science, Dongshin University, Naju, Cheonnam, Korea 520-714 \\ ${ }^{2,3}$ Division of Mathematics and Informational Statistics, and Nanoscale Science and Technology Institute, \\ Wonkwang University, Iksan, Chonbuk, Korea 570-749
}

\begin{abstract}
We introduce the notions of interval-valued fuzzy prime ideals, interval-valued fuzzy completely prime ideals and intervalvalued fuzzy weakly completely prime ideals. And we give a characterization of interval-valued fuzzy ideals and establish relationships between interval-valued fuzzy completely prime ideals and interval-valued fuzzy weakly completely prime ideals.
\end{abstract}

Key Words: interval-valued fuzzy set, interval-valued fuzzy subring. interval-valued fuzzy ideal, interval-valued fuzzy prime ideal, interval-valued fuzzy completely prime ideal, interval-valued fuzzy weakly completely prime ideal.

\section{Introduction and Preliminaries}

In 1975, Zadeh[11] introduced the concept of intervalvalued fuzzy sets as a generalization of fuzzy sets introduced by himself[10]. After then, Biswas[1] applied the notion of interval-valued fuzzy sets to group theory. Moreover, Gorzalczany[4] applied it to a method of inference in approximate reasoning, and Montal and Samanta[8] applied it to topology. Recently, Hur et al.[5] introduced the concept of an interval-valued fuzzy relations and obtained some of it's properties. Also, Choi et al.[3] applied it to topology in the sense of $\breve{S}$ ostak, Kang and Hur[7], and Kang[6] applied it to algebra.

In this paper, we introduce the notions of interval-valued fuzzy prime ideals, interval-valued fuzzy completely prime ideals and interval-valued fuzzy weakly completely prime ideals. And we give a characterization of interval-valued fuzzy ideals and establish relationships between intervalvalued fuzzy completely prime ideals and interval-valued fuzzy weakly completely prime ideals.

Now, we will list some basic concepts and well-known results which are needed in the later sections.

Let $D(I)$ be the set of all closed subintervals of the unit interval $I=[0,1]$. The elements of $D(I)$ are generally denoted by capital letters $M, N, \cdots$, and note that $M=\left[M^{L}, M^{U}\right]$, where $M^{L}$ and $M^{U}$ are the lower and the upper end points respectively. Especially, we denoted,

Manuscript received May. 23, 2012; revised Sep. 21, 2012; accepted Sep. 24, 2012.

${ }^{3}$ Corresponding Author : pklim@wonkwang.ac.kr 2000 Mathematics Subject Classification. 54A40.

(C)The Korean Institute of Intelligent Systems. All rights reserved. $\mathbf{0}=[0,0], \mathbf{1}=[1,1]$, and $\boldsymbol{a}=[a, a]$ for every $a \in(0,1)$. We also note that

(i) $(\forall M, N \in D(I))\left(M=N \Leftrightarrow M^{L}=N^{L}, M^{U}=\right.$ $\left.N^{U}\right)$ $N^{U}$ (ii)

(ii) $(\forall M, N \in D(I))\left(M \leq N \Leftrightarrow M^{L} \leq N^{L}, M^{U} \leq\right.$ For every $M \in D(I)$, the complement of $M$, denoted by $M^{c}$, is defined by $M^{c}=1-M=\left[1-M^{U}, 1-M^{L}\right]($ See [8]).

Definition 1.1 [8, 11]. A mapping $A: X \rightarrow D(I)$ is called an interval-valued fuzzy set (in short, IVS) in $X$ and is denoted by $A=\left[A^{L}, A^{U}\right]$. Thus for each $x \in X$, $A(x)=\left[A^{L}(x), A^{U}(x)\right]$, where $A^{L}(x)\left[\right.$ resp. $\left.A^{U}(x)\right]$ is called the lower [resp. upper] end point of $x$ to $A$. For any $[a, b] \in D(I)$, the interval-valued fuzzy set $A$ in $X$ defined by $A(x)=[a, b]$ for each $x \in X$ is denoted by $\widetilde{[a, b}]$ and if $a=b$, then the IVS $\widetilde{a, b}]$ is denoted by simply $\widetilde{a}$. In particular, $\tilde{0}$ and $\tilde{1}$ denote the interval-valued fuzzy empty set and the interval-valued fuzzy whole set in $X$, respectively.

We will denote the set of all IVSs in $X$ as $D(I)^{X}$. It is clear that set $A=\left[A^{L}, A^{U}\right] \in D(I)^{X}$ for each $A \in I^{X}$.

Definition 1.2 [8]. Let $A, B \in D(I)^{X}$ and let $\left\{A_{\alpha}\right\}_{\alpha \in \Gamma} \subset$ $D(I)^{X}$. Then
(a) $A \subset B$ iff $A^{L} \leq B^{L}$ and $A^{U} \leq B^{U}$.
(b) $A=B$ iff $A \subset B$ and $B \subset A$.
(c) $A^{c}=\left[1-A^{U}, 1-A^{L}\right]$.
(d) $A \cup B=\left[A^{L} \vee B^{L}, A^{U} \vee B^{U}\right]$.
(d) $\bigcup_{\alpha \in \Gamma} A_{\alpha}=\left[\bigvee_{\alpha \in \Gamma} A_{\alpha}^{L}, \bigvee_{\alpha \in \Gamma} A_{\alpha}^{U}\right]$
(e) $A \cap B=\left[A^{L} \wedge B^{L}, A^{U} \wedge B^{U}\right]$. 
$(\mathrm{e})^{\prime} \bigcap_{\alpha \in \Gamma} A_{\alpha}=\left[\bigwedge_{\alpha \in \Gamma} A_{\alpha}^{L}, \bigwedge_{\alpha \in \Gamma} A_{\alpha}^{U}\right]$

Result 1.A [8, Theorem 1]. Let $A, B, C \in D(I)^{X}$ and let $\left\{A_{\alpha}\right\}_{\alpha \in \Gamma} \subset D(I)^{X}$. Then
(a) $\tilde{0} \subset A \subset \tilde{1}$.
(b) $A \cup B=B \cup A, A \cap B=B \cap A$.
(c) $A \cup(B \cup C)=(A \cup B) \cup C$, $A \cap(B \cap C)=(A \cap B) \cap C$.
(d) $A, B \subset A \cup B, A \cap B \subset A, B$.
(e) $A \cap\left(\bigcup_{\alpha \in \Gamma} A_{\alpha}\right)=\bigcup_{\alpha \in \Gamma}\left(A \cap A_{\alpha}\right)$.
(f) $A \cup\left(\bigcap_{\alpha \in \Gamma} A_{\alpha}\right)=\bigcap_{\alpha \in \Gamma}\left(A \cup A_{\alpha}\right)$.
(g) $(\tilde{0})^{c}=\tilde{1},(\tilde{1})^{c}=\tilde{0}$.
(h) $\left(A^{c}\right)^{c}=A$.
(i) $\left(\bigcup_{\alpha \in \Gamma} A_{\alpha}\right)^{c}=\bigcap_{\alpha \in \Gamma} A_{\alpha}^{c},\left(\bigcap_{\alpha \in \Gamma} A_{\alpha}\right)^{c}=\bigcup_{\alpha \in \Gamma} A_{\alpha}^{c}$.

Definition 1.3 [7]. Let $A$ be an IVS in a set $X$ and let $[\lambda, \mu] \in D(I)$. Then the set $A^{[\lambda, \mu]}=\left\{x \in X: A^{L}(x) \geq\right.$ $\lambda$ and $\left.A^{U}(x) \geq \mu\right\}$ is called a $[\lambda, \mu]$-level subset of $A$.

Definition 1.4 [8]. Let $[\lambda, \mu] \in D(I)$. Then an intervalvalued fuzzy point(in short, IVP) $x_{[\lambda, \mu]}$ of $X$ is the IVS in $X$ defined as follows : For each $y \in X$,

$$
x_{[\lambda, \mu]}(y)= \begin{cases}{[\lambda, \mu],} & \text { if } y=x \\ \tilde{0}, & \text { otherwise }\end{cases}
$$

In this case, $x$ is called the support of $x_{[\lambda, \mu]}$ and, $\lambda$ and $\mu$ are called the value and nonvalue of $x_{[\lambda, \mu]}$, respectively. In particular, if $\lambda=\mu$, then it is also denoted by $x_{\boldsymbol{\lambda}}$. An IVP $x_{M}$ is said to belong to an IVS $A$ in $X$, denoted by $x_{M} \in A$ if $M^{L} \leq A^{L}(x)$ and $M^{U} \leq A^{U}(x)$.

It is clear that $A=\cup_{x_{M} \in A} x_{M}$ and $x_{M} \in A$ if and only if $x_{M^{L}} \in A^{L}$ and $x_{M^{U}} \in A^{U}$, for each $A \in P(I)^{X}$.

We will denote the set of all IVPs in $X$ as $\operatorname{IVP}(X)$.

The following is the immediate result of Definition 1.2 and 1.4.

Theorem 1.5. Let $A, B \in D(I)^{X}$. Then $A \subset B$ if and only if for each $x_{M} \in \operatorname{IVP}(\mathrm{X}), x_{M} \in A$ implies $x_{M} \in B$.

Definition 1.6 [7]. Let $(X, \cdot)$ be a groupoid and let $A, B \in$ $D(I)^{X}$. Then the interval-valued fuzzy product of $A$ and $B, A \circ B$ is defined as follows : For each $x \in X$,

$$
\begin{aligned}
& A \circ B(x) \\
= & \begin{cases}{\left[\bigvee_{x=y z}\left(A^{L}(y) \wedge B^{L}(z)\right),\right.} & \text { orherwise. } \\
\left.\bigvee_{x=y z}\left(A^{U}(y) \wedge B^{U}(z)\right)\right], & \text { if } x=y z ;\end{cases}
\end{aligned}
$$

Result 1.B [7, Proposition 3.2]. Let $(X, \cdot)$ be a groupoid, let "o" be the same as above, let $x_{M}, y_{N} \in \operatorname{IVP}(\mathrm{X})$ and let $A, B \in D(I)^{X}$. Then
(a) $x_{M} \circ y_{N}=(x y)_{M \cap N}$.
(b) $A \circ B=\bigcup_{x_{M} \in A, y_{N} \in B} x_{M} \circ y_{N}$.

Definition 1.7 [1]. Let $G$ be a group and let $A \in D(I)^{G}$. Then $A$ is called an interval-valued fuzzy subgroup (in short, IVG) of $G$ if it satisfies the following conditions :

(a) $A^{L}(x y) \geq A^{L}(x) \wedge A^{L}(y)$ and $A^{U}(x y) \geq A^{U}(x) \wedge$ $A^{U}(y)$ for any $x, y \in G$.

(b) $A^{L}\left(x^{-1}\right) \geq A^{L}(x)$ and $A^{U}\left(x^{-1}\right) \geq A^{U}(x)$ for each $x \in G$.

We will denote the set of all IVGs as IVG(G).

Result 1.C [1, Proposition 3.1]. Let $A$ be an IVG of a group $G$ with identity $e$. Then $A\left(x^{-1}\right)=A(x)$ and $A^{L}(x) \geq A^{L}(e), A^{U}(x) \geq A^{U}(e)$ for each $x \in G$.

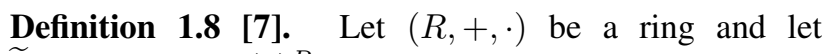
$\widetilde{0} \neq A \in D(I)^{R}$. Then $A$ is called an intervalvalued fuzzy subring (in short, IVR) of $R$ if it satisfies following conditions :

(a) $A$ is an IVG with respect to the operation " + ".

(b) $A^{L}(x y) \geq A^{L}(x) \wedge A^{L}(y)$ and $A^{U}(x y) \geq$ $A^{U}(x) \wedge A^{U}(y)$ for any $x, y \in R$.

We will denote the set of all IVRs as IVR(R).

\section{Interval-valued fuzzy ideals}

Definition 2.1 [7]. Let $A$ be a non-empty IVR of a ring $R$. Then $A$ is called an :

(i) interval-valued fuzzy left ideal (in short, IVLI) of $R$ if $A^{L}(x y) \geq A^{L}(y)$ and $A^{U}(x y) \geq A^{U}(y)$ for any $x, y \in$ $R$.

(ii) interval-valued fuzzy right ideal (in short, IVRI) of $R$ if $A^{L}(x y) \geq A^{L}(x)$ and $A^{U}(x y) \geq A^{U}(x)$ for any $x, y \in R$.

(iii) interval-valued fuzzy ideal (in short, IVRI) of $R$ if it is an IVLI and an IVRI of $R$.

We will denote the set of all IVRIs [resp. IVLIs and IVIs] of $R$ as IVRI(R) [resp. IVLI(R) and IVI(R) ].

Result 2.A [7, Proposition 6.6]. Let $R$ be a ring. Then $A$ is an ideal [resp. a left ideal and a right ideal ] of $R$ if and any of $\left[\chi_{A}, \chi_{A}\right] \in \operatorname{IVI}(\mathrm{R})$ [resp. IVLI(R) and IVRI(R)].

Result 2.B [7, Proposition 6.5]. Let $R$ be a ring and let $\tilde{0} \neq A \in D(I)^{R}$. Then $A \in \operatorname{IVI}(\mathrm{R})[$ resp. IVLI(R) and $\operatorname{IVRI}(\mathrm{R})]$ if and only if for any $x, y \in R$, 
(i) $A^{L}(x-y) \geq A^{L}(x) \wedge A^{L}(y)$ and $A^{U}(x-y) \geq$ $A^{U}(x) \wedge A^{U}(y)$.

(ii) $A^{L}(x y) \geq A^{L}(x) \wedge A^{L}(y)$ and $A^{U}(x y) \geq$ $A^{U}(x) \wedge A^{U}(y)$ [resp. $A^{L}(x y) \geq A^{L}(y)$ and $A^{U}(x y) \geq A^{U}(y), A^{L}(x y) \geq A^{L}(x)$ and $\left.A^{U}(x y) \geq \bar{A}^{U}(x)\right]$.

Lemma 2.2. Let $R$ be a ring and let $A, B \in D(I)^{R}$.

(a) If $A, B \in \operatorname{IVLI}(\mathrm{R})$ [resp. IVRI(R) and IVI(R)], then $A \cap B \in \operatorname{IVLI}(\mathrm{R})$ [resp. IVRI(R) and IVI(R)].

(b) If $A \in \operatorname{IVRI}(\mathrm{R})$ and $B \in \operatorname{IVLI}(\mathrm{R})$, then $A \circ B \subset A \cap B$.

Proof. (a) Suppose $A, B \in \operatorname{IVLI}(\mathrm{R})$ and let $x, y \in R$. Then

$$
\begin{aligned}
& (A \cap B)^{L}(x-y) \\
= & A^{L}(x-y) \wedge B^{L}(x-y) \\
\geq & \left(A^{L}(x) \wedge A^{L}(y)\right) \wedge\left(B^{L}(x) \wedge B^{L}(y)\right) \\
= & (A \cap B)^{L}(x) \wedge(A \cap B)^{L}(y) .
\end{aligned}
$$

Similarly, we have $(A \cap B)^{U}(x-y) \geq(A \cap B)^{U}(x) \wedge$ $(A \cap B)^{U}(y)$. Also

$$
\begin{aligned}
& (A \cap B)^{L}(x y) \\
= & A^{L}(x y) \wedge B^{L}(x y) \\
\geq & A^{L}(y) \wedge B^{L}(y) \quad(\text { Since } A, B \in \mathrm{IVLI}(\mathrm{R})) \\
= & (A \cap B)^{L}(y) .
\end{aligned}
$$

Similarly, we have $(A \cap B)^{U}(x y) \geq(A \cap B)^{U}(y)$. Hence, by Result 2.B, $A \cap B \in \operatorname{IVLI}(\mathrm{R})$. Similarly, we can easily see the rest.

(b) Let $x \in G$ and suppose $A \circ B(x)=[0,0]$. Then there is nothing to show. Suppose $A \circ B(x) \neq[0,0]$. Then $A \circ B(x)=\left[\bigvee_{x=y z}\left(A^{L}(y) \wedge B^{L}(z)\right), \bigvee_{x=y z}\left(A^{U}(y) \wedge\right.\right.$ $\left.\left.B^{U}(z)\right)\right]$. Since $A \in \operatorname{IVRI}(\mathrm{R})$ and $B \in \operatorname{IVLI}(\mathrm{R})$,

$A^{L}(y) \leq A^{L}(y z)=A^{L}(x), A^{U}(y) \leq A^{U}(y z)=A^{U}(x)$

and

$B^{L}(z) \leq B^{L}(y z)=B^{L}(x), B^{U}(z) \leq B^{U}(y z)=B^{U}(x)$.

Thus

$$
\begin{aligned}
(A \circ B)^{L}(x) & =\bigvee_{x=y z}\left(A^{L}(y) \wedge B^{L}(z)\right) \\
& \leq A^{L}(x) \wedge B^{L}(x)=(A \cap B)^{L}(x) .
\end{aligned}
$$

Similarly, we have $(A \circ B)^{U}(x) \leq(A \cap B)^{U}(x)$. Hence $A \circ B \subset A \cap B$. This completes the proof.

A ring $R$ is said to be regular if for each $a \in R$ there exists an $x \in R$ such that $a=a x a$.
Result 2.C [2, Theorem 9.4]. A ring $R$ is regular if and only if $J M=J \cap M$ for each right ideal $J$ and left ideal $M$ of $R$.

Theorem 2.3. A ring $R$ is regular if and only if for each $A \in \mathrm{IVRI}(\mathrm{R})$ and each $B \in \mathrm{IVLI}(\mathrm{R}), A \circ B=A \cap B$.

Proof. $(\Rightarrow)$ : Suppose $R$ is regular. From Lemma 2.2(b), it is clear that $A \circ B \subset A \cap B$. Thus it is sufficient to show that $A \cap B \subset A \circ B$. Let $a \in R$. Then, by the hypothesis, there exists an $x \in R$ such that $a=a x a$. Thus $A^{L}(a)=A^{L}(a x a) \geq A^{L}(a x) \geq A^{L}(a)$ and $A^{U}(a)=$ $A^{U}(a x a) \geq A^{U}(a x) \geq A^{U}(a)$. So $A(a x)=A(a)$.

On the other hand,

$$
\begin{aligned}
(A \circ B)^{L}(a) & =\bigvee_{a=y z}\left(A^{L}(y) \wedge B^{L}(z)\right) \\
& \geq A^{L}(a x) \wedge B^{L}(a) \quad(\text { Since } a=a x a) \\
& =A^{L}(a) \wedge B^{L}(a)=(A \cap B)^{L}(a) .
\end{aligned}
$$

Similarly, we have $(A \circ B)^{U}(a) \geq(A \cap B)^{U}(a)$. Thus $A \cap B \subset A \circ B$. Hence $A \circ B=A \cap B$.

$(\Leftarrow)$ : Suppose the necessary condition holds. Let $J$ and $M$ be right and left ideals of $R$, respectively. Then, by Result 2.A, $\left[\chi_{J}, \chi_{J}\right] \in \operatorname{IVRI}(\mathrm{R})$ and $\left[\chi_{M}, \chi_{M}\right] \in \operatorname{IVLI}(\mathrm{R})$. Let $a \in J \cap M$ and let $A=\left[\chi_{J}, \chi_{J}\right], B=\left[\chi_{M}, \chi_{M}\right]$. Then, by the hypothesis, $(A \circ B)(a)=(A \cap B)(a)=[1,1]$. Thus

$$
\begin{aligned}
(A \circ B)^{L}(a) & =\bigvee_{a=a_{1} a_{2}}\left(A^{L}\left(a_{1}\right) \wedge B^{L}\left(a_{2}\right)\right) \\
& =\bigvee_{a=a_{1} a_{2}}\left(\chi_{J}\left(a_{1}\right) \wedge \chi_{M}\left(a_{2}\right)\right) \\
& =1 .
\end{aligned}
$$

Similarly, we have $(A \circ B)^{U}(a)=1$. So there exist $b_{1}, b_{2} \in R$ such that $\chi_{J}\left(b_{1}\right)=1$ and $\chi_{M}\left(b_{2}\right)=1$ with $a=b_{1} b_{2}$. Thus $a \in J M$, i.e., $J \cap M \subset J M$. Since $J M \subset J \cap M, J M=J \cap M$. Hence, by Result 2.C, $R$ is regular. This completes the proof.

\section{Interval-valued fuzzy prime ideals}

Definition 3.1. Let $P$ be an IVI of a ring $R$. Then $P$ is said to be prime if $P$ is not a constant mapping and for any $A, B \in \mathrm{IVI}(\mathrm{R}), A \circ B \subset P$ implies either $A \subset P$ or $B \subset P$.

We will denote the set of all interval-valued fuzzy prime ideals of $R$ as IVPI(R).

Theorem 3.2. Let $J$ be an ideal of a ring $R$ such that $J \neq R$. Then $J$ is a prime ideal of $R$ if and only if 
$\left[\chi_{J}, \chi_{J}\right] \in \mathrm{IVPI}(\mathrm{R})$.

Proof. $(\Rightarrow)$ : Suppose $J$ is a prime ideal of $R$ and let $P=\left[\chi_{J}, \chi_{J}\right]$. Since $J \neq R, P$ is not a constant mapping on $R$. Assume that there exist $A, B \in \operatorname{IVI}(\mathrm{R})$ such that $A \circ B \subset P$ and $A \not \subset P$ and $B \not \subset P$. Then there exist $x, y \in R$ such that

$$
A^{L}(x)>P^{L}(x)=\chi_{J}(x), A^{U}(x)>P^{U}(x)=\chi_{J}(x)
$$

and

$$
B^{L}(y)>P^{L}(y)=\chi_{J}(y), B^{U}(y)>P^{U}(y)=\chi_{J}(y) .
$$

Thus $A^{L}(x) \neq 0, A^{U}(x) \neq 0$ and $B^{L}(y) \neq 0, B^{U}(y) \neq 0$. But $\chi_{J}(x)=0$ and $\chi_{J}(y)=0$. So $x \notin J$ and $y \notin J$. Since $J$ is a prime ideal of $R$, by the process of the proof of Theorem 2 in [9], there exist an $r \in R$ such that $x r y \notin J$. Let $a=x r y$. Then clearly, $\chi_{J}(a)=0$. Thus

$$
A \circ B(a)=[0,0] \text {. }
$$

On the other hand,

$$
\begin{aligned}
(A \circ B)^{L}(a) & =\bigvee_{a=c d}\left(A^{L}(c) \wedge B^{L}(d)\right) \\
& \geq A^{L}(x) \wedge B^{L}(r y) \quad(\text { Since } a=x r y) \\
& =A^{L}(x) \wedge B^{L}(y)(\text { Since } B \in \mathrm{IVI}(\mathrm{R})) \\
& >0 .\left(\text { Since } A^{L}(x) \neq 0 \text { and } B^{L}(y) \neq 0\right)
\end{aligned}
$$

Similarly, we have $(A \circ B)^{U}(a)>0$. Then $A \circ B(a) \neq \widetilde{0}$. This contradicts (3.1). So $P$ satisfies the second condition of Definition 3.1. Hence $P=\left[\chi_{J}, \chi_{J}\right] \in \operatorname{IVPI}(\mathrm{R})$.

$(\Leftarrow)$ : Suppose $P=\left[\chi_{J}, \chi_{J}\right] \in \operatorname{IVPI}(\mathrm{R})$. Since $P$ is not a constant mapping on $R, J \neq R$. Let $A$ and $B$ be two ideals of $R$ such that $A B \subset J$. Let $\widetilde{A}, \widetilde{B} \in \operatorname{IVI}(\mathrm{R})$. Consider the product $\widetilde{A} \circ \widetilde{B}$. Let $x \in R$.

Suppose $\widetilde{A} \circ \widetilde{B}(x)=[0,0]$. Then clearly $\widetilde{A} \circ \widetilde{B} \subset P$.

Suppose $\widetilde{A} \circ \widetilde{B}(x) \neq[0,0]$. Then $(\widetilde{A} \circ \widetilde{B})^{L}(x)=$ $\bigvee_{x=y z}\left(\chi_{A}(y) \wedge \chi_{B}(z)\right) \neq 0$. Similarly, we have $(\widetilde{A} \circ \widetilde{B})^{U}(x) \neq 0$. Thus there exist $y, z \in R$ with $x=y z$ such that $\chi_{A}(y) \neq 0$ and $\chi_{B}(z) \neq 0$. So $\chi_{A}(y)=1$ and $\chi_{B}(z)=1$. This implies $y \in A$ and $z \in B$. Thus $x=y z \in A B \subset J$. So $\chi_{J}(x)=1$. It follows that $\widetilde{A} \circ \widetilde{B} \subset P$. Since $P \in \operatorname{IVPI}(\mathrm{R})$, either $\widetilde{A} \subset P$ or $\widetilde{B} \subset P$. Thus either $A \subset J$ or $B \subset J$. Hence $J$ is a prime ideal of $R$. This completes the proof.

Proposition 3.3. Let $P$ be an interval-valued fuzzy prime ideals of a ring $R$ and let $R_{P}=\{x \in R: P(x)=P(0)\}$. Then $R_{P}$ is a prime ideal of $R$.

Proof. Let $x, y \in R_{P}$. Then $P(x)=P(0)$ and $P(y)=$ $P(0)$. Thus $P^{L}(x-y) \geq P^{L}(x) \wedge P^{L}(y)=P^{L}(0)$. Similarly, we have $P^{U}(x-y) \geq P^{U}(0)$. Since $P \in \operatorname{IVI}(\mathrm{R})$,

$$
P^{L}(0)=P^{L}(0(x-y)) \geq P^{L}(x-y) .
$$

Similarly, we have $P^{U}(0) \geq P^{U}(x-y)$. So $x-y \in R_{P}$. Now let $r \in R$ and let $x \in R_{P}$. Then

$P^{L}(r x) \geq P^{L}(x)=P^{L}(0)$ and $P^{U}(r x) \geq P^{U}(x)=P^{U}(0)$.

By Result 1.C, $P(r x)=P(0)$. So $r x \in R_{P}$. Similarly we have $x r \in R_{P}$. Hence $R_{P}$ is an ideal of $R$.

Let $J$ and $M$ be two ideals of $R$ such that $J M \subset$ $R_{P}$. We define two mappings $A, B: R \rightarrow D(I)$ by $A=P(0)\left[\chi_{J}, \chi_{J}\right]$ and $B=P(0)\left[\chi_{M}, \chi_{M}\right]$, respectively, where $P(0)\left[\chi_{J}, \chi_{J}\right]=\left[P^{L}(0) \chi_{J}, P^{U}(0) \chi_{J}\right]$. Then we can easily prove that $A, B \in \mathrm{IVI}(\mathrm{R})$. Let $x \in R$.

Suppose $A \circ B(x)=[0,0]$. Then $A \circ B \subset P$.

Suppose $A \circ B(x) \neq[0,0]$. Then $(A \circ B)^{L}(x)=$ $\bigvee_{x=y z}\left(A^{L}(y) \wedge B^{L}(z)\right)=\bigvee_{x=y z}\left(P^{L}(0) \chi_{J}(y) \wedge\right.$ $\left.P^{L}(0) \chi_{M}(z)\right) \neq 0$. Similarly, we have $(A \circ B)^{U}(x) \neq 0$. Thus there exist $y, z \in R$ with $x=y z$ such that

$$
P^{L}(0) \chi_{J}(y) \wedge P^{L}(0) \chi_{M}(z) \neq 0
$$

and

$$
P^{U}(0) \chi_{J}(y) \wedge P^{U}(0) \chi_{M}(z) \neq 0 .
$$

So $\chi_{J}(y)=1$ and $\chi_{M}(z)=1$. Thus $y \in J$ and $z \in M$, i.e., $x=y z \in J M \subset R_{P}$. So $P(x)=P(0)$, i.e., $A \circ B \subset P$. Since $P \in \operatorname{IVPI}(\mathrm{R})$ and $A, B \in \mathrm{IVI}(\mathrm{R})$, either $A \subset P$ or $B \subset P$. Suppose $A \subset P$. Then $P(0)\left[\chi_{J}, \chi_{J}\right] \subset P$. Assume that $J \subset R_{P}$. Then there exists an $a \in J$ such that $a \notin R_{P}$. Thus $P(a) \neq P(0)$. By Result 1.C, $P^{L}(a)<P^{L}(0)$ and $P^{U}(a)<P^{U}(0)$. Then $A^{L}(a)=P^{L}(0) \chi_{J}(a)=P^{L}(0)>P^{L}(a)$. Similarly, we have $A^{U}(a)>P^{U}(a)$. This contradicts the assumption that $A \subset P$. So $J \subset R_{P}$. By the similar arguments, we can show that if $B \subset P$, then $M \subset R_{P}$. Hence $R_{P}$ is a prime ideal of $R$. This completes the proof.

Remark 3.4. Let $P \in \operatorname{IVI}(\mathbb{Z})$. Then, by Proposition 3.3, $R_{P}$ is an ideal of $\mathbb{Z}$. Hence there exists an integer $n \geq 0$ such that $R_{P}=n \mathbb{Z}$.

Proposition 3.5. Let $P \in \operatorname{IVI}(\mathbb{Z})$ with $R_{P}=n \mathbb{Z} \neq(0)$. Then $P$ can take at most $r$ values, where $r$ is the number of distinct positive divisors of $n$.

Proof . Let $a \in \mathbb{Z}$ and let $d=(a, n)$. Then there exist $r, s \in \mathbb{Z}$ such that $d=a r+n s$. Thus

$P^{L}(d)=P^{L}(a r+n s) \geq P^{L}(a r) \wedge P^{L}(n s) \geq P^{L}(a) \wedge P^{L}(n)$.

Similarly, we have $P^{U}(d) \geq P^{U}(a) \wedge P^{U}(n)$. Since $n \in$ $R_{P}=n \mathbb{Z}$, by Result 1.C,

$P^{L}(n)=P^{L}(0) \geq P^{L}(a)$ and $P^{U}(n)=P^{U}(0) \geq P^{U}(a)$.

Thus $P^{L}(d) \geq P^{L}(a)$ and $P^{U}(d) \geq P^{U}(a)$. Since $d$ is a divisor of $a$, there exists a $t \in \mathbb{Z}$ such that $a=d t$. Then 


$$
\begin{aligned}
& \quad P^{L}(a)=P^{L}(d t) \geq P^{L}(d) \text { and } P^{U}(a)=P^{U}(d t) \geq \\
& P^{U}(d) .
\end{aligned}
$$

So $P(a)=P(d)$. Moreover, by Result 1.C, $P(x)=P(-x)$ for each $x \in R$. Hence for each $a \in \mathbb{Z}$ there exists a positive divisor $d$ of $n$ such that $P(a)=P(d)$. This completes the proof.

The following result gives a complete characterization of interval-valued fuzzy prime ideals of $\mathbb{Z}$ :

Theorem 3.6. Let $P \in \operatorname{IVPI}(\mathbb{Z})$ with $\mathbb{Z}_{P} \neq(0)$. Then $P$ has two distinct values. Conversely, if $P \in D(I)^{\mathbb{Z}}$ such that $P(n)=\left[\lambda_{1}, \mu_{1}\right]$ when $p \mid n$ and $P(n)=\left[\lambda_{2}, \mu_{2}\right]$ when $p \nmid n$, where $p$ is a fixed prime, $\lambda_{1}>\lambda_{2}$ and $\mu_{1}>\mu_{2}$, then $P \in \operatorname{IVPI}(\mathbb{Z})$ with $\mathbb{Z}_{P} \neq(0)$.

Proof. Suppose $P \in \operatorname{IVPI}(\mathbb{Z})$ with $\mathbb{Z}_{P}=n \mathbb{Z} \neq(0)$. Then, by Proposition 3.3, $\mathbb{Z}_{P}$ is a prime ideal of $\mathbb{Z}$. Thus $n$ is a prime integer. Since $n$ has two distinct positive integers, by Proposition $3.5, P$ has at most two distinct values. On the other hand, an interval-valued fuzzy prime ideals cannot be a constant mapping. Hence $P$ has two distinct values.

Conversely, let $P$ be an IVS in $\mathbb{Z}$ satisfying the given conditions. Let $a, b \in \mathbb{Z}$.

Case(i): Suppose $p \mid(a-b)$. Then $P(a-b)=\left[\lambda_{1}, \mu_{1}\right]$. Thus $\lambda_{1}=P^{L}(a-b) \geq P^{L}(a) \wedge P^{L}(b)$ (Since $\left.\lambda_{1}>\lambda_{2}\right)$ and $\mu_{1}=P^{U}(a-b) \geq P^{U}(a) \wedge P^{U}(b)\left(\right.$ Since $\left.\mu_{1}>\mu_{2}\right)$.

Case(ii): Suppose $p \nmid(a-b)$. Then $p \nmid a$ or $p \nmid b$. Thus either $P(a)=\left[\lambda_{2}, \mu_{2}\right]$ or $P(b)=\left[\lambda_{2}, \mu_{2}\right]$. So $\lambda_{2}=$ $P^{L}(a-b) \geq P^{L}(a) \wedge P^{L}(b)$ and $\mu_{2}=P^{U}(a-b) \geq$ $P^{U}(a) \wedge P^{U}(b)$.

Case(iii): Suppose $p \mid a b$. Then clearly $P^{L}(a b) \geq$ $P^{L}(b)$ and $P^{U}(a b) \geq P^{U}(b)$.

Case(iv): Suppose $p \nmid a b$. Then $p \nmid a$ and $p \nmid b$. Thus $P^{L}(a b) \geq P^{L}(b)$ and $P^{U}(a b) \geq P^{U}(b)$. Consequently, by Result 1.C, $P \in \operatorname{IVI}(\mathbb{Z})$ with $\mathbb{Z}_{P}=p \mathbb{Z} \neq(0)$. Moreover, by the similar arguments of the proof of Proposition 3.2, we can see that $P \in \operatorname{IVPI}(\mathbb{Z})$. This completes the proof.

Proposition 3.7. Let $R$ be a ring with 1 . If every IVI of $R$ has finite values, then $R$ is a Noetherian ring.

Proof . Let $\left\{J_{i}\right\}_{i \in \mathbb{Z}^{+}}$be a sequence of ideals of $R$ such that $J_{1} \subset J_{2} \subset J_{3} \subset \cdots$ and let $J=\bigcup_{i \in \mathbb{Z}^{+}} J_{i}$. Then clearly $J$ is an ideal of $R$. We define a mapping $P: R \rightarrow D(I)$ as follows : For each $x \in R$,

$$
P(x)= \begin{cases}\mathbf{0}, & \text { if } x \notin J ; \\ {\left[\frac{1}{i_{1}}, \frac{1}{i_{1}}\right],} & \text { if } x \in J .\end{cases}
$$

where $i_{1}=$ minimum of $i$ such that $x \in J_{i}$. Then it is clear that $P \in \operatorname{IVI}(\mathrm{R})$ from the definition of $P$. Moreover, we can easily see that $P \in \mathrm{IVI}(\mathrm{R})$. If the chain does not terminate, then $P$ takes infinitely many values. This contradicts the hypothesis. Thus the chain terminates. Hence $R$ is a Noetherian ring. This completes the proof.

Proposition 3.8. Let $A: \mathbb{Z} \rightarrow D(I)$ be the mapping such that

(a) $A(x)=A(-x)$ for each $x \in \mathbb{Z}$.

(b) $A^{L}(x+y) \geq A^{L}(x) \wedge A^{L}(y)$ and $A^{U}(x+y) \geq$ $A^{U}(x) \wedge A^{U}(y)$ for any $x, y \in \mathbb{Z}$.

If there exists a non-zero integer $m$ such that $A(m)=A(0)$, then $A$ can take at most finitely many values.

Proof. It is clear that $A \in D(I)^{\mathbb{Z}}$ from the definition of $A$. Moreover, we can easily show that $A \in \operatorname{IVI}(\mathbb{Z})$ such that $\mathbb{Z}_{A} \neq(0)$. Hence, by Proposition 3.5, $A$ can take at most finitely many values.

\section{Interval-valued fuzzy completely prime ideals}

Definition 4.1. Let $P$ be an IVI of a ring $R$. Then $P$ is called an interval-valued fuzzy completely prime ideals(in short, IVCPI) of $R$ if it satisfies the following conditions :

(a) $P$ is not a constant mapping.

(b) For any $x_{M}, y_{N} \in \mathrm{IVP}(\mathrm{R}), x_{M} \circ y_{N} \in P$ implies either $x_{M} \in P$ or $y_{N} \in P$.

We will denote the set of all IVCPIs of $R$ as $\operatorname{IVCPI}(G)$.

Proposition 4.2. (a) Let $R$ be a ring. Then $\operatorname{IVCPI}(R) \subset$ $\operatorname{IVPI}(R)$.

(b) Let $R$ be a commutative ring. Then $\operatorname{IVPI}(R) \subset$ $\operatorname{IVCPI}(R)$. Hence $\operatorname{IVCPI}(R)=\operatorname{IVPI}(R)$.

Proof . (a) Let $P \in \operatorname{IVCPI}(R)$ and let $A, B \in \operatorname{IVI}(\mathrm{R})$ such that $A \circ B \subset P$. Suppose $A \not \subset P$. Then, by Theorem 1.5, there exists an $x_{[\lambda, \mu]} \in \operatorname{IVP}(R)$ such that $x_{[\lambda, \mu]} \in P$ but $x_{[\lambda, \mu]} \notin P$. Let $y_{[t, s]} \in B$. Then, by Result 1.B(a), $x_{[\lambda, \mu]} \circ y_{[t, s]}=(x y)_{[\lambda \wedge t, \mu \wedge s]}$. On the other hand,

$$
\begin{aligned}
P^{L}(x y) & \geq(A \circ B)^{L}(x y) \geq A^{L}(x) \wedge B^{L}(y) \\
& =\lambda \wedge t=\left(x_{[\lambda, \mu]} \circ y_{[t, s]}\right)^{L}(x y) .
\end{aligned}
$$

Similarly, we have $P^{U}(x y) \geq\left(x_{[\lambda, \mu]} \circ y_{[t, s]}\right)^{U}(x y)$.

Let $z \in R$ such that $x \neq x y$. Then clearly $\left[x_{[\lambda, \mu]} \circ\right.$ $\left.y_{[t, s]}\right](z)=[0,0]$. Thus $x_{[\lambda, \mu]} \circ y_{[t, s]} \in P$. Since $P \in$ $\operatorname{IVCPI}(R), x_{[\lambda, \mu]} \in P$ or $y_{[t, s]} \in P$. Since $x_{[\lambda, \mu]} \notin P$, $y_{[t, s]} \in P$. So, by Theorem 1.5, $B \subset P$. Hence $P \in$ IVPI(R).

(b) Let $P \in \operatorname{IVPI}(\mathrm{R})$ and let $x_{[\lambda, \mu]}, y_{[t, s]} \in \operatorname{IVP}(\mathrm{R})$ such that $x_{[\lambda, \mu]} \circ y_{[t, s]} \in P$. Then $\left(x_{[\lambda, \mu]} \circ y_{[t, s]}\right)^{L}(x y) \leq$ $P^{L}(x y)$ and $\left(x_{[\lambda, \mu]} \circ y_{[t, s]}\right)^{U}(x y) \leq P^{U}(x y)$. 
Thus, by Result 1.B(a),

$$
\lambda \wedge t \leq P^{L}(x y) \text { and } \mu \wedge s \leq P^{U}(x y) .
$$

We define two mappings $A, B: R \rightarrow D(I)$ as follows : For each $z \in R$,

$$
A(z)= \begin{cases}{[\lambda, \mu],} & \text { if } z \in(x) \\ {[0,0],} & \text { otherwise }\end{cases}
$$

and

$$
B(z)= \begin{cases}{[t, s],} & \text { if } z \in(y) \\ {[0,0],} & \text { otherwise }\end{cases}
$$

where $(x)$ is the ideal generated by $x$. Then clearly $A, B \in$ $D(I)^{R}$ from the definitions of $A$ and $B$. It is easily seen that if $z$ is not expressible in the form $z=u v$ for some $u \in(x)$ and $v \in(y)$, then $A \circ B(z)=[0,0]$. Suppose there exist $u \in(x)$ and $v \in(y)$ such that $z=u v$. Then

$$
(A \circ B)^{L}(z)=\bigvee_{z=u v, u \in(x), v \in(y)}\left(A^{L}(u) \wedge B^{L}(v)\right)=\lambda \wedge t
$$

and

$$
(A \circ B)^{U}(z)=\bigvee_{z=u v, u \in(x), v \in(y)}\left(A^{U}(u) \wedge B^{U}(v)\right)=\mu \wedge s .
$$

Since $R$ is commutative and $u \in(x)$, there exist $n \in \mathbb{Z}$ and $b \in R$ such that $u=n x+x b$. Since $v \in(y)$, there exist $m \in \mathbb{Z}$ and $c \in R$ such that $v=m y+y c$. Since $R$ is commutative, $u v=(n x+x b)(m y+y c)=x y d+$ $m n x y$ for some $d \in R$. Then

$$
\begin{aligned}
P^{L}(u v) & \geq P^{L}(x y) & & (\text { Since } P \in \mathrm{IVI}(\mathrm{R})) \\
& \geq \lambda \wedge t . & & (\mathrm{By}(4.1))
\end{aligned}
$$

Similarly, we have that $P^{U}(u v) \geq P^{U}(x y) \geq \mu \wedge s$. Thus $z_{[\lambda \wedge t, \mu \wedge s]}=u_{[\lambda, \mu]} \circ v_{[t, s]} \in P$. So, in all, $A \circ B \subset P$. On the other hand, from the definitions of $A$ and $B$, we can easily prove that $A, B \in \operatorname{IVI}(\mathrm{R})$. Since $P \in \operatorname{IVPI}(\mathrm{R})$, either $A \subset P$ or $B \subset P$. Thus either $x_{[\lambda, \mu]} \in P$ or $y_{[t, s]} \in P$. Hence $P \in \operatorname{IVCPI}(R)$. This completes the proof.

Proposition 4.3. Let $P$ be a non-constant IVI of a ring $R$.

(a) If $P$ is an IVPI [resp. IVCPI] of $R$, then

(i) $R_{P}$ is a prime [resp. completely prime] ideal of $R$.

(ii) $\operatorname{Im} P$ consists of exactly two points of $D(I)$.

(b) If $P(0)=[1,1]$ and $P$ satisfies the conditions (i) and (ii), then $P \in \operatorname{IVPI}(R)[\operatorname{resp} \operatorname{IVCPI}(R)]$.

Proof - (a) We shall confirm our proof to the case of interval-valued fuzzy prime ideals. An analogous proof can be given by for interval-valued fuzzy completely prime ideals. Suppose $P \in \operatorname{IVPI}(\mathrm{R})$. Then, by Proposition 3.3, $R_{P}$ is a prime ideal of $R$. Assume that $\operatorname{Im} P$ contains more than two values. Then there exist $x, y \in R \backslash R_{P}$ such that $P(x) \neq P(y)$. Suppose without loss of generality that $P^{L}(x)<P^{L}(y)$ and $P^{U}(x)<P^{U}(y)$. Since $P \in \mathrm{IVI}(\mathrm{R})$ and $A(y) \neq A(0)$, by Result 1.C, $P^{L}(x)<$ $P^{L}(y)<P^{L}(0)$ and $P^{U}(x)<P^{U}(y)<P^{U}(0)$. Let $[\lambda, \mu],[t, s] \in D(I)$ be chosen such that

$$
P^{L}(x)<\lambda<P^{L}(y)<t<P^{L}(0)
$$

and

$$
P^{U}(x)<\mu<P^{U}(y)<s<P^{U}(0) .
$$

Let $(x)$ and $(y)$ denote respectively the ideals generated by $x$ and $y$. We define two mappings $A, B: R \rightarrow D(I)$ as follows: $A=\left[\lambda \chi_{(x)}, \mu \chi_{(x)}\right]$ and $B=\left[t \chi_{(y)}, s \chi_{(y)}\right]$. Then it is easily seen that $A, B \in \operatorname{IVI}(\mathrm{R})$ from the definitions of $A$ and $B$. Let $z \in R$ which cannot be expressed in the from $z=u v$ for $u \in(x)$ and $v \in(y)$. Then $A \circ B(z)=[0,0]$. Thus $A \circ B \subset P$. Now let $z \in R$. Suppose there exist $u \in(x)$ and $v \in(y)$ such that $z=u v$ for some $u \in(x)$ and $v \in(y)$. Then

$$
(A \circ B)^{L}(z)=\bigvee_{z=u v, u \in(x), v \in(y)}\left(A^{L}(u) \wedge B^{L}(v)\right)=\lambda \wedge t=\lambda .
$$

Similarly, we have $(A \circ B)^{U}(z)=\mu$. Since $u \in(x)$, there exist $m \in \mathbb{Z}$ and $r_{i} \in R(i=1,2,3,4)$ such that $u=$ $m x+r_{1} x+x r_{2}+r_{3} x r_{4}$. Similarly, there exist $n \in \mathbb{Z}$ and $s_{i} \in R(i=1,2,3,4)$ such that $v=n y+s_{1} y+y s_{2}+s_{3} y s_{4}$. Since $P \in \mathrm{IVI}(\mathrm{R})$, by Result 1.C,

$$
P^{L}(z)=P^{L}(u v) \geq P^{L}(x) \wedge P^{L}(y)>\lambda
$$

and

$$
P^{U}(z)=P^{U}(u v) \geq P^{U}(x) \wedge P^{U}(y)>\mu .
$$

Thus $(A \circ B)^{L}(z) \leq P^{L}(z)$ and $(A \circ B)^{U}(z) \leq P^{U}(z)$. So $A \circ B \subset P$. Since $P \in \operatorname{IVPI}(\mathrm{R})$, either $A \subset P$ or $B \subset P$. Then either $A^{L}(x)=\lambda \leq P^{L}(x), A^{U}(x)=\mu \leq$ $P^{U}(x)$ or $B^{L}(y)=t \leq P^{L}(y), B^{U}(y)=s \leq P^{U}(y)$. This contradicts (4.2). Hence $\operatorname{Im} P$ consists of exactly two points of $D(I)$.

(b) Suppose $P(0)=[1,1]$ and $P$ satisfies the conditions (i) and (ii). Then, by the similar arguments of proof of Theorem 3.2, we can see that $P \in \operatorname{IVPI}(\mathrm{R})$. This completes the proof.

Corollary 4.3. Let $P$ be an interval-valued fuzzy completely prime ideal of a ring $R$. Then for any $x, y \in R$, $P(x y)=\left[P^{L}(x) \wedge P^{L}(y), P^{U}(x) \wedge P^{U}(y)\right]$.

Remark 4.4. Proposition 4.3 generalizes Proposition 3.5 .

Definition 4.5. Let $A$ be a non-constant IVI of a ring $R$. Then $A$ is called an interval-valued fuzzy weakly completely prime ideal of $R$ if for any 


$$
x, y \in R, A(x y)=\left[A^{L}(x) \wedge A^{L}(y), A^{U}(x) \wedge A^{U}(y)\right] .
$$

The following is the immediate result of Definitions 4.1 and 4.5 .

Proposition 4.6. Let $A$ be an interval-valued fuzzy weakly completely prime ideal of a ring $R$. Then for each $[\lambda, \mu] \in D(I), x_{[\lambda, \mu]} \circ y_{[t, s]} \in A$ implies that either $x_{[\lambda, \mu]} \in A$ or $y_{[t, s]} \in A$. Furthermore, for each $[\lambda, \mu] \in D(I)$ such that $\lambda+\mu \leq 1, \lambda<A^{L}(0)$ and $\mu<A^{U}(0), A^{[\lambda, \mu]}$ is a completely prime ideal of $R$. In particular, $A^{[0,0]}$ is a completely prime ideal of $R$. Conversely if for each $[\lambda, \mu] \in D(I), A^{[\lambda, \mu]}$ is a completely prime ideal then $A$ is an interval-valued fuzzy weakly completely prime ideal.

The following is the example that an interval-valued fuzzy weakly completely prime ideal need not be an interval-valued fuzzy completely prime ideal.

Example 4.7. Let $R=\mathbb{Z} \times \mathbb{Z}$, let $S=\{0\} \times \mathbb{Z}$ and let $T=(2) \times \mathbb{Z}$. We define a mapping $A: R \rightarrow D(I)$ as follows : For each $x \in R$,

$$
A(x)= \begin{cases}{[1,1],} & \text { if } x \in S ; \\ \left(\frac{1}{2}, \frac{1}{3}\right), & \text { if } x \in T \backslash S ; \\ {[0,0],} & \text { if } x \in R \backslash T .\end{cases}
$$

Then clearly $A \in D(I)^{R}$ from the definition of $A$. Moreover, we can easily show that $A$ is an interval-valued fuzzy weakly completely prime ideal but, by Proposition 4.2, $A$ is not an interval-valued fuzzy weakly completely prime ideal.

\section{References}

[1] R.Biswas, "Rosenfeld's fuzzy subgroups with interval-valued membership functions," Fuzzy set and systems, vol. 63, pp. 87-90, 1995.

[2] D.M.Burton, "A First Course in Rings and Ideals"(Addition-Wesley, 1970).

[3] J.Y.Choi, S.R.Kim and K.Hur, "Interval-valued smooth topological spaces," Honam Math.J., vol. 32, pp. 711-738, 2010.
[4] M.B. Gorzalczany, "A method of inference in approximate reasoning based on interval-valued fuzzy sets," Fuzzy Sets and Systems, vol. 21, pp. 1-17, 1987.

[5] K.Hur, J.G.Lee and J.Y.Choi, "Interval-valued fuzzy relations," J.Korean Institute of Intelligent systems, vol. 19, pp. 425-432, 2009.

[6] H.W.Kang, "Interval-valued fuzzy subgroups and homomorphisms," Honam Math. J., vol. 33, 2011.

[7] K.Hur and H.W.Kang, "Interval-valued fuzzy subgroups and rings," Honam Math.J., vol. 32, pp. 593$617,2010$.

[8] T.K.Mondal and S.K.Samanta, "Topology of intervalvalued fuzzy sets," Indian J.Pure Appl.Math., vol. 30, pp. 20-38, 1999.

[9] T.K.Mukherjee and M.K.Sen, "On fuzzy ideals of a ring," Fuzzy sets and Systems, vol. 21, pp. 98-104, 1987.

[10] L.A.Zadeh, "Fuzzy sets," Inoform. and Centrol, vol. 8, pp. 338-353, 1965.

[11] L.A.Zadeh, "The concept of a linguistic variable and its application to approximate reasoning I," Inform.Sci, vol. 8, pp. 199-249, 1975.

\section{Keon Chang Lee}

Professor in Dongshin University

His research interests are Category Theory, Hyperspace and Topology.

E-mail : kclee@dsu.ac.kr

\section{Kul Hur}

Professor in Wonkwang University

His research interests are Category Theory, Hyperspace and Topology.

E-mail : kulhur@wonkwang.ac.kr

\section{Pyung Ki Lim}

Professor in Wonkwang University

His research interests are Category Theory, Hyperspace and Topology.

E-mail : pklim@wonkwang.ac.kr 